\title{
DETERMINING THE UNBIASED ESTIMATOR OF THE POPULATION GEOMETRIC MEASURES OF VARIATION ABOUT THE MEAN
}

\author{
Troon John Benedict ${ }^{1}$, Obere Almadi ${ }^{2}$, Karanjah Anthony ${ }^{3}$, Alilah Anekeya David ${ }^{4}$ \\ 1Department of Mathematics and Physical Science, Maasai Mara University, Narok, Kenya \\ 2Department of Economics, Maasai Mara University, Narok, Kenya \\ 3Department of Mathematics, Multimedia University, Nairobi, Kenya \\ 4Department of Mathematics and Statistics, Masinde Muliro University of Science and Technology, Kakamega, Kenya
}

Email address:

troonbenedict@gmail.com (T. J. Benedict),aobere@mmarau.ac.ke, karanjah@mmarau.ac.ke (K. Anthony), aliladavid2010@gmail.com (A. A. David)

\begin{abstract}
Geometric Measures of Variation about the mean is a measure that uses the geometric averaging technique to average the deviations from the mean. From previous studies, it has been determined that the measure is more precise in estimating the average variation about the mean than the existing measures of variation about the mean. Given that the technique is a newly introduced technique of estimating the average variation about the mean, the actual sample estimator for the measure is still unknown, as a result, the study aimed at determining the unbiased estimator for the population geometric measure. The study used a mathematical estimation technique to determine the unbiased estimator among the existing possible estimators as it assumed a simple random sampling without replacement technique. The study determined that the unbiased estimator of the population estimator was the sample estimator which did not allow one degree of freedom.
\end{abstract}

Key Words: Estimator, Parameter, Unbiasedness, Sampling

\section{Introduction}

Geometric Measures of Variation about the mean is a measure that uses the geometric averaging technique to average the deviations from the mean $[15,16]$. Unlike other measures of variation about the mean such as mean deviation, variance, and standard deviation [1,2,5]. The measure has been determined to be more efficient in estimating the average variation about the mean than the existing measures because of various reasons such as; unlike mean deviation, the measure average absolute products and not sums $[9,11,13]$, which makes the estimates more precise based on the algebraic number theory on absolute numbers which shows that;

Given $a \neq 0, b \neq 0$ and $a, b \in \mathfrak{R}$ by the definition of an absolute number which is a transformation such that $|\bullet|: \mathfrak{R} \rightarrow+\mathfrak{R}(7)$.
1. $|a b|=|a||b|$
2. $|a+b| \leq|a|+|b|$

This shows that the averaging of deviation products by the geometric measures are more precise as illustrated by condition (1), however, the estimates given by mean deviation are not precise 
because there is a slight difference between the actual values and the estimates as illustrated by the triangular inequality in condition $(2)[8,12]$.

The geometric measure gives estimates of average variation about the mean of same units of measurement as the original dataset, unlike, variance which gives squared units of measurement for the estimates of average variation about the mean [1,12]). Lastly, the measure uses geometric averaging which is nonresponsive to outliers and skewed datasets, as a result, the measure is not affected by outliers and skewed datasets, unlike standard deviation. All these shows that geometric measure is a more superior measure of variation about the mean $[1,2,12]$.

In most research work, due to various factors such as cost, time constrain, impossibility in accessing all respondents in the study area among others, most researchers always opt to carry out sample surveys as opposed to complete enumeration of all respondents in the population, as a result, most researchers dependents on the sample estimates to make inferential conclusions regarding the population. Therefore, a researcher is always required to pick an estimator that would precisely estimate the population parameter during the estimation process $[3,8]$.

As newly formulated measures of variation about the mean, the unbiased sample estimator of the population parameter still unknown. As a result, the aim of this study was to determine the unbiased estimator of the population geometric measure of variation, which will assist in the precise estimation of the measure for various samples $[15,16]$.

\section{Methods}

\section{Possible estimators}

In determining the unbiased estimator of the population geometric measure of variation about the mean, the study compared two possible estimators of the population geometric measures mathematically [8].

Consider a population vector of observations $V=\left[v_{1}, v_{2}, v_{3}, \ldots, v_{N}\right]$. We can define the mean of the population vector $\bar{V}$ as

$\bar{V}=\frac{\sum_{i=1}^{N} v_{i}}{N}$

Further, define the $\mathrm{i}^{\text {th }}$ population deviation $D_{i}$ as

$D_{i}=v_{i}-\bar{V}$

Therefore, the population deviation vector will be given by $D=\left[D_{1}, D_{2}, D_{3}, \ldots, D_{N}\right]$. Now assume that all the observations in the population $v_{i} \neq \bar{V}$, therefore, all $D_{i} \neq 0$. Hence, by definition, the geometric measure of variation about the mean $\mathrm{G}$ for the population will be given by $[15,16]$ 
$G=\left\{\begin{array}{cc}\sqrt[N]{\prod_{i=1}^{N}\left|D_{i}\right|} & \forall D_{i} \neq 0 \\ 0 & \forall D_{i}=0\end{array}\right.$

Using natural logarithms, Equation (3) can be simplified as

$$
G=\left\{\begin{array}{cl}
\exp \left(\frac{\sum_{i=1}^{N} \ln \left|D_{i}\right|}{N}\right) & \forall D_{i} \neq 0 \\
0 & \forall D_{i}=0
\end{array}\right.
$$

Consider a random sample vector of size $\mathrm{n}$ from the above population vector and a dummy weight $k_{i}$ such that [3]

$$
k_{i}=\left\{\begin{array}{l}
1 \\
0
\end{array}\right.
$$

where $k_{i}=1$ when the coefficient observation in the population $v_{i}$ is selected into the sample and $k_{i}=0$ when the coefficient observation in the population $v_{i}$ is not selected into the sample. Based on this, the sample mean $\bar{v}$ is defined by

$$
\bar{v}=\frac{\sum_{i=1}^{N} k_{i} v_{i}}{n}=\frac{1}{n}\left(\sum_{i=1}^{N} k_{i} v_{i}\right)
$$

Based on the above sample, we can estimate the population geometric measure of variation using two methods. First, we can average the sample average variation about the mean using geometric average as follows $[15,16]$.

Define the $\mathrm{i}^{\text {th }}$ deviation from the sample mean $\bar{v}$ as

$$
d_{i}=v_{i}-\bar{v}
$$

The sample geometric measure of variation about the mean $g_{r}$ will be given by

$$
g_{r}=\left\{\begin{array}{cc}
\sqrt[n]{\prod_{i=1}^{N}\left|d_{i}\right|^{k_{i}}} & \forall d_{i} \neq 0 \\
0 & \forall d_{i}=0
\end{array}\right.
$$

The above estimate can be simplified using logarithm as 


$$
g_{r}=\left\{\begin{array}{cc}
\exp \left(\frac{\sum_{i=1}^{N} k_{i} \ln \left|d_{i}\right|}{n}\right) & \forall d_{i} \neq 0 \\
0 & \forall d_{i}=0
\end{array}\right.
$$

Secondly, we can estimate the population geometric measure of variation about the mean by allowing a 1 degree of freedom in the sample estimation process, this will result into a new measure of variation about the mean $g_{q}$ which is given by $[15,16]$

$$
g_{q}=\left\{\begin{array}{cc}
\sqrt[n-1]{\prod_{i=1}^{N}\left|d_{i}\right|^{k_{i}}} & \forall d_{i} \neq 0 \\
0 & \forall d_{i}=0
\end{array}\right.
$$

Equation [10] can be simplified using logarithm to obtain

$$
g_{q}=\left\{\begin{array}{cc}
\exp \left(\frac{\sum_{i=1}^{N} k_{i} \ln \left|d_{i}\right|}{n-1}\right) & \forall d_{i} \neq 0 \\
0 & \forall d_{i}=0
\end{array}\right.
$$

The above two methods are all possible estimators of the population geometric measure. As a result, the study aims at proving which of the two estimators is an unbiased estimator of the population geometric measure.

Assuming a homogenous population distribution, the study assumes a simple random sampling technique without replacement during the estimation process of the population parameter using either of the two methods $[14,17]$.

\section{Results}

\section{Theorem}

Given the population geometric measure $\mathrm{G}$, an estimator $\mathrm{g}$ of the parameter is said to be unbiased by definition if [4].

$$
E(g)=G
$$

Based on this theorem, if any of the two estimators $g_{r}$ or $g_{q}$ is an unbiased estimator of the population parameter $\mathrm{G}$ then by definition of an unbiased estimator

$$
E\left(g_{r}\right)=G
$$

or 
$E\left(g_{q}\right)=G$

\section{Proof}

We can determine which one of these two estimators is the true unbiased estimator of the parameter $G$ as follows;

Starting with $g_{r}$ considering a random sample of size $\mathrm{n}$, from a population $V=\left[V_{1}, V_{2}, V_{3}, \ldots, V_{N}\right]$. The sample vector $\mathrm{v}$ selected at random using simple random sampling without replacement from the population is given by $v=\left\{k_{i} V_{i} \mid i=1,2,3,4, \ldots N\right\}$ where $k_{i}$ is a dummy weight such that $[3,14$, 17]

$k_{i}=\left\{\begin{array}{l}1 \\ 0\end{array}\right.$

where $k_{i}=1$ when the coefficient observation in the population $V_{i}$ is selected into the sample and $k_{i}=0$ when the coefficient observation in the population $V_{i}$ is not selected into the sample. The sample mean is given by $[3,6]$

$$
\bar{\nu}=\frac{\sum_{i=1}^{N} k_{i} V_{i}}{n}
$$

The $\mathrm{i}^{\text {th }}$ deviation from the mean is defined by

$d_{i}=V_{i}-\bar{v}$

The geometric measure of variation about the mean $g_{r}$ is therefore given by

$$
g_{r}=\left\{\begin{array}{cc}
\sqrt[n]{\prod_{i=1}^{N}\left|d_{i}\right|^{k_{i}}} & \forall d_{i} \neq 0 \\
0 & \forall d_{i}=0
\end{array}\right.
$$

This can be simplified as

$$
g_{r}=\left\{\begin{array}{cc}
\exp \left(\frac{\sum_{i=1}^{N} k_{i} \ln \left|d_{i}\right|}{n}\right) & \forall d_{i} \neq 0 \\
0 & \forall d_{i}=0
\end{array}\right.
$$

Given that the population geometric measure $\mathrm{G}$ is defined by 
$G=\left\{\begin{array}{cc}\exp \left(\frac{\sum_{i=1}^{N} k_{i} \ln \left|D_{i}\right|}{n}\right) & \forall D_{i} \neq 0 \\ 0 & \forall D_{i}=0\end{array}\right.$

Then if $g_{r}$ is an unbiased estimator of $\mathrm{G}$ then

$E\left(g_{r}\right)=G=\left\{\begin{array}{cc}\exp \left(\frac{\sum_{i=1}^{N} k_{i} \ln \left|D_{i}\right|}{n}\right) & \forall d_{i} \neq 0 \\ 0 & \forall d_{i}=0\end{array}\right.$

Now given the unique nature of exponential such that

$\exp (K)=\exp (R)$, iff $K=R$

Then if $\ln \left(g_{r}\right)$ is an unbiased estimator of $\ln (G)$, then $g_{k}$ is an unbiased estimator of G. Now, if $\ln \left(g_{r}\right)$ is an unbiased estimator of $\ln (G)$ then

$E\left(\ln \left(g_{r}\right)\right)=\ln (G)$

By definition

$\ln \left(g_{r}\right)=\frac{\sum_{i=1}^{N} k_{i} \ln |d|_{i}}{n}=\frac{1}{n}\left(\sum_{i=1}^{N} k_{i} \ln \left|d_{i}\right|\right)$

Then

$E\left(\ln \left(g_{r}\right)\right)=E\left(\frac{1}{n} \sum_{i=1}^{N} k_{i} \ln \left|d_{i}\right|\right)=\frac{1}{n} \sum_{i=1}^{N} E\left(k_{i} \ln \left|d_{i}\right|\right)$

It can be shown that

$E\left(\ln \left|d_{i}\right|\right)=\ln \left|D_{i}\right|$

This is because

$E\left(\ln \left|d_{i}\right|\right)=\ln \left(E\left|d_{i}\right|\right)=\ln \left(E\left|V_{i}-\bar{v}\right|\right)=\ln \left(\left|E\left(V_{i}\right)-E(\bar{v})\right|\right)$

Because the sample mean $\bar{v}$ is an unbiased estimator of the population mean, therefore $=\ln \left|V_{i}-\bar{V}\right|=\ln \left|D_{i}\right|$ therefore, 


$$
E\left(\ln \left(g_{r}\right)\right)=E\left(\frac{1}{n} \sum_{i=1}^{N} k_{i} \ln \left|d_{i}\right|\right)=\frac{1}{n} \sum_{i=1}^{N} E\left(k_{i} \ln \left|d_{i}\right|\right)=\frac{1}{n} \sum_{i=1}^{N} \ln \left|D_{i}\right| E\left(k_{i}\right)
$$

Now, given that $k_{i}$ is a binary dummy variable, it can be seen that $k_{i}$ is Bernoulli distributed with probability mass function

$$
P\left(k_{i}\right)=\left\{\begin{array}{cc}
p^{k_{i}}(1-p)^{1-k_{i}} & k_{i}=0,1 \\
0 & \text { otherwise }
\end{array}\right.
$$

Therefore, the expected value of $k_{i}$ by definition is $\mathrm{p}$. we can determine the actual value of $\mathrm{p}$ through obtaining the maximum likelihood estimator of $\mathrm{p}$ from the probability mass function as follows [4];

Let $\mathrm{L}$ be the likelihood function of the probability mass function of $k_{i}$ which is given as

$$
L=\prod_{i=1}^{N} p^{k_{i}}(1-p)^{1-k_{i}}=p^{\sum_{i=1}^{N} k_{i}}(1-p)^{N-\sum_{i=1}^{N} k_{i}}
$$

Now we get the log-likelihood function, which is given by

$$
\begin{aligned}
& \ln L=\ln p^{\sum_{i=1}^{N} k_{i}}(1-p)^{N-\sum_{i=1}^{N} k_{i}}=\sum_{i=1}^{N} k_{i} \ln p+N-\sum_{i=1}^{N} k_{i} \ln (1-p) \\
& \ln L=\sum_{i=1}^{N} k_{i} \ln p+N \ln (1-p)-\sum_{i=1}^{N} k_{i} \ln (1-p)
\end{aligned}
$$

Now, differentiating Equation (21) with respect to $\mathrm{p}$ in order to maximize the estimator we get that

$$
\begin{aligned}
& \frac{\partial \ln L}{\partial p}=\sum_{i=1}^{N} k_{i}\left(\frac{1}{p}\right)+N\left(\frac{-1}{1-p}\right)-\sum_{i=1}^{N} k_{i}\left(\frac{-1}{1-p}\right) \\
& \frac{\partial \ln L}{\partial p}=\frac{\sum_{i=1}^{N} k_{i}}{p}-\frac{N}{1-p}+\frac{\sum_{i=1}^{N} k_{i}}{1-p}=\frac{\sum_{i=1}^{N} k_{i}(1-p)-N p+\sum_{i=1}^{N} k_{i}(p)}{p-p^{2}} \\
& \frac{\partial \ln L}{\partial p}=\frac{\sum_{i=1}^{N} k_{i}-\sum_{i=1}^{N} k_{i}(p)-N p+\sum_{i=1}^{N} k_{i}(p)}{p-p^{2}} \\
& \frac{\partial \ln L}{\partial p}=\frac{\sum_{i=1}^{N} k_{i}-N p}{p-p^{2}}
\end{aligned}
$$

Equating the above Equation (22) to zero we have that 


$$
\begin{aligned}
& \frac{\partial \ln L}{\partial p}=\frac{\sum_{i=1}^{N} k_{i}-N p}{p-p^{2}}=0 \\
& \therefore \sum_{i=1}^{N} k_{i}-N p=0 \\
& \therefore N p=\sum_{i=1}^{N} k_{i} \\
& p=\frac{\sum_{i=1}^{N} k_{i}}{N}
\end{aligned}
$$

But there are $\mathrm{n} k_{i}$ terms which are equivalent to 1 with the rest being equivalent to 0 , therefore, it can be shown that

$$
\sum_{i=1}^{N} k_{i}=n
$$

Hence, the maximum likelihood estimator of $p=\frac{n}{N}$. Therefore

$$
E\left(k_{i}\right)=\frac{n}{N}
$$

Hence, going back to Equation (18) we can now show that

$$
E\left(\ln \left(g_{r}\right)\right)=\frac{1}{n} \sum_{i=1}^{N} \ln \left|D_{i}\right| E\left(k_{i}\right)=\frac{1}{n} \sum_{i=1}^{N} \ln \left|D_{i}\right| \frac{n}{N}=\frac{1}{n} \bullet \frac{n}{N} \sum_{i=1}^{N} \ln \left|D_{i}\right|=\frac{1}{N} \sum_{i=1}^{N} \ln \left|D_{i}\right|=\ln (G)
$$

Now because

$$
E\left(\ln \left(g_{r}\right)\right)=\ln (G)
$$

Introducing exponential both sides

$$
\begin{aligned}
& \exp \left(E\left(\ln \left(g_{r}\right)\right)\right)=\exp (\ln (G)) \\
& E\left(\exp \left(\ln \left(g_{r}\right)\right)\right)=G \\
& E\left(g_{r}\right)=G
\end{aligned}
$$

Hence, the estimator $g_{r}$ if an unbiased estimator of the population parameter $\mathrm{G}$.

Second, Consider the estimator $g_{q}$ which is given by the function 


$$
g_{q}=\left\{\begin{array}{cc}
\exp \left(\frac{\sum_{i=1}^{N} k_{i} \ln \left|d_{i}\right|}{n-1}\right) & \forall d_{i} \neq 0 \\
0 & \forall d_{i}=0
\end{array}\right.
$$

Similar to $g_{r}$, if $g_{q}$ is an unbiased estimator of $\mathrm{G}$ then

$$
\begin{aligned}
& E\left(g_{q}\right)=G \\
& E\left(g_{q}\right)=G=\left\{\begin{array}{cc}
\exp \left(\frac{\sum_{i=1}^{N} k_{i} \ln \left|D_{i}\right|}{n-1}\right) & \forall d_{i} \neq 0 \\
0 & \forall d_{i}=0
\end{array}\right.
\end{aligned}
$$

Given the unique character of exponentiation illustrated in Equation (13), then if Equation (26) is true, then

$$
\begin{aligned}
& E\left(\ln \left(g_{q}\right)\right)=\ln (G) \\
& E\left(\ln \left(g_{q}\right)\right)=E\left(\frac{1}{n-1} \sum_{i=1}^{N} k_{i} \ln \left|d_{i}\right|\right)=\frac{1}{n} \sum_{i=1}^{N} E\left(k_{i} \ln \left|d_{i}\right|\right)
\end{aligned}
$$

But according to Equation (18)

$\ln \left|V_{i}-\bar{V}\right|=\ln \left|D_{i}\right|$

And according to Equation (24)

$$
E\left(k_{i}\right)=\frac{n}{N}
$$

Therefore,

$$
\begin{aligned}
& \quad E\left(\ln \left(g_{q}\right)\right)=E\left(\frac{1}{n-1} \sum_{i=1}^{N} k_{i} \ln \left|d_{i}\right|\right)=\frac{1}{n-1} \sum_{i=1}^{N} \ln \left|D_{i}\right| \frac{n}{N}=\frac{n}{N n-N} \sum_{i=1}^{N} \ln \left|D_{i}\right| \neq \frac{1}{N} \sum_{i=1}^{N} \ln \left|D_{i}\right| \\
& E\left(\ln \left(g_{q}\right)\right) \neq \ln (G) \\
& \therefore \exp \left(E\left(\ln \left(g_{q}\right)\right)\right) \neq \exp (\ln (G)) \\
& \therefore E\left(\exp \left(\ln \left(g_{q}\right)\right)\right) \neq \exp (\ln (G)) \\
& \therefore E\left(g_{q}\right) \neq G
\end{aligned}
$$

Therefore, the estimator $g_{q}$ is a biased estimator of the population geometric measure G. 


\section{Conclusion}

In conclusion, considering a simple random sample without replacement, the unbiased estimator of the population geometric measure is given by the estimator which does not allow one degree of freedom during the estimation process of the population parameter. The unbiased estimator is given by

$$
g_{r}=\left\{\begin{array}{cc}
\exp \left(\frac{\sum_{i=1}^{N} k_{i} \ln \left|d_{i}\right|}{n}\right) & \forall d_{i} \neq 0 \\
0 & \forall d_{i}=0
\end{array}\right.
$$

\section{Recommendation}

When estimating the average variation about the mean for a sample using the geometric measure, one should use the above estimator $g_{r}$ because it is unbiased and more precise in estimating the average variation about the mean for a sample.

\section{References}

[1]. Ahn, S., \& Fessler, J. A., (2003). Standard Errors of Mean, Variance, and Standard Deviation Estimators. EECS Department. The University of Michigan. U.S.A

[2]. Altman, D. G., \& Bland, J. M. (2005). Standard deviations and standard errors. BMJ Volume 331.

[3]. Banning, R, Camstra, A. and Knottnerus, P. (2012). Sampling theory: Sampling design and estimation methods. Statistics Netherlands. Henri Faasdreef 312. 2492 JP The Hague.

[4]. Bensoussan, A., Bertrand, P. and Brouste, A. (2015). Future Perspectives in Risk Models and Finance. International Series in Operations Research \& Management Science 211. Springer International Publishing Switzerland DOI 10.1007/978-3-319-07524-2_1

[5]. Bhardwaj, A., (2013). Comparative Study of Various Measures of Dispersion. Journal of Advances in Mathematics. Vol 1, No 1.

[6]. Camstra, A. and Nieuwenbroek, N.J. (2002), Syllabus for the sampling theory course. CBS-Academie, Voorburg

[7]. Clark, P. L., (2012). Number Theory: A Contemporary Introduction. Available at http://math.uga.edu/ pete/4400FULL.pdf

[8]. Deshpande, S., Gogtay, N. J., Thatte, U. M., (2016). Measures of Central Tendency and Dispersion. Journal of the Association of Physicians of India.Vol. 64. July 2016.

[9]. Hu, S., (2010). Simple Mean, Weighted Mean, or Geometric Mean?. Presented at the 2010 ISPA/SCEA Joint Annual Conference and Training Workshop

[10]. Manikandan, S., (2016). Measures of dispersion. Journal of Pharmacology and Pharmacotherapeutics. October-December 2011. Vol 2. Issue 4

[11]. Mindlin, D., (2011). On the Relationship between Arithmetic and Geometric Returns. Cdi Advisors Research. LLC.

[12]. Roberson, Q. M., Sturman, M. C., \& Simons, T. L., (2007). Does the Measure of Dispersion Matter in Multilevel Research? A Comparison of the Relative Performance of 
Dispersion Indexes. Cornell University School of Hotel Administration. The Scholarly Commons.

[13]. Roenfeldt, K., (2018). Better than average: Calculating Geometric Means Using SAS. Henry. M. Foundation for the Advancement of Military Medicine.

[14]. Singh, A. and Masuku, M. (2014). Sampling Techniques \& Determination of Sample Size in Applied Statistics Research: An Overview. International Journal of Economics, Commerce and Management. Vol. II, Issue 11.

[15]. Troon, J. B., Karanjah, A., and Alilah, A. D.(2019). Modeling Geometric Measure of Variation About the Population Mean. American Journal of Theoretical and Applied Statistics. Vol. 8, No. 5, pp. 179-184. doi: 10.11648/j.ajtas.20190805.13

[16]. Troon, J. B., Karanjah, A., and Alilah, A. D.(2019). Empirical Comparison of Relative Precision of Geometric Measure of Variation about the Mean and Standard Deviation. International Journal of Recent Engineering Research and Development (IJRERD). Volume 04 - Issue 10. pp. 01-12.

[17]. Yates, F. and Grundy, P.M. (1953), Selection without replacement from within strata with probability proportional to size. Journal of the Royal Statistical Society B, 15, 253261. 